\title{
Clinical Trial Team Curriculum Vitae
}

National Cancer Institute

\section{Source}

National Cancer Institute. Clinical Trial Team Curriculum Vitae. NCI Thesaurus. Code C125441.

A summary of educational and work experience qualifications and eligibility of study team members and Trial Team Members affiliated with the Site, Sponsor and Sponsor partner(s). Excludes Principal Investigator or Sub-Investigator. 